\title{
Fabrication of Metal Alloy-Deposited Flexible MWCNT Buckypaper for Thermoelectric Applications
}

\author{
Jih-Hsin Liu, ${ }^{1,2}$ Hsin-Yuan Miao, ${ }^{1,2}$ Saravanan Lakshmanan, \\ Li-Chih Wang, ${ }^{2,3}$ and Ren-Hui Tsai ${ }^{1}$ \\ ${ }^{1}$ Department of Electrical Engineering, Tunghai University, 181 Taichung Harbor Road, Section 3, Taichung 40704, Taiwan \\ ${ }^{2}$ Tunghai Green Energy Development and Management Institute, Tunghai University, 181 Taichung Harbor Road, Section 3, \\ Taichung 40704, Taiwan \\ ${ }^{3}$ Department of Industrial Engineering and Enterprise Information, Tunghai University, 181 Taichung Harbor Road, Section 3 , \\ Taichung 40704, Taiwan
}

Correspondence should be addressed to Hsin-Yuan Miao; kenymiao@thu.edu.tw

Received 31 March 2013; Revised 10 September 2013; Accepted 27 September 2013

Academic Editor: Sulin Zhang

Copyright (C) 2013 Jih-Hsin Liu et al. This is an open access article distributed under the Creative Commons Attribution License, which permits unrestricted use, distribution, and reproduction in any medium, provided the original work is properly cited.

We report the fabrication of a flexible network of multiwall carbon nanotubes (MWCNTs) known as buckypaper (BP) for thermoelectric (TE) applications. A thermal evaporation method was used to deposit TE metal alloys onto the BP. The TE properties were improved primarily by increasing the Seebeck coefficient values (50 and $75 \mu \mathrm{V} / \mathrm{K}$ ) and the electrical conductivity by approximately $10000 \mathrm{~S} / \mathrm{m}$. High-temperature resistivity studies were performed to confirm the semiconductivity of buckypaper. Variations in resistivity were observed to be the result of the metal alloys coated on the BP surface. We also demonstrated that a substantial increase in the Seebeck coefficient values can be obtained by connecting 3 and 5 layers of metal-deposited BP in series, thereby enhancing the TE efficiency of MWCNT-based BP for application in thermoelectric devices.

\section{Introduction}

Recently, a considerable amount of effort has been devoted to developing thermoelectric (TE) devices that are capable of generating electricity from heat. TE devices that conserve heat energy should be cost-efficient and possess a high thermoelectric figure of merit [1]. Good TE materials must have a high Seebeck coefficient, which enhances thermoelectricity, low electrical resistivity, which minimizes Joule heating, and low thermal conductivity, which sustains a large temperature gradient [2]. The performance of a TE material is determined by its dimensionless figure of merit $Z T=S^{2} T / \rho \kappa$, where $S$, $\rho$, and $\kappa$ are the Seebeck coefficient, electrical resistivity, and thermal conductivity of the materials, respectively.

Previous studies have focused on semimetallic nanomaterials such as $\mathrm{Bi}, \mathrm{Sb}, \mathrm{Te}$, and $\mathrm{Bi}-\mathrm{Sb}$ alloys because of their promising thermoelectric properties [3-5]. $\mathrm{Bi}_{2} \mathrm{Te}_{3}$-based alloys are known to be a state-of-the-art material currently available for TE applications [6]. Recently, Zhang et al. [7] reported that the Seebeck coefficient of $\mathrm{Bi}_{2} \mathrm{Te}_{3}$ can be improved by incorporating single wall carbon nanotubes (SWCNTs) into the matrix. Furthermore, highly doped SWCNTs can be used to tune $\mathrm{Bi}_{2} \mathrm{Te}_{3}$ from $n$ - to $p$-type. Despite their excellent thermoelectric properties, these materials exhibit poor mechanical property, which complicates the fabrication process, and can cause reliability problems in their application, especially in miniature TE modules.

Recently, there has been considerable focus on macroscopic assemblies of various carbon nanotubes (CNTs) such as buckypaper (BP), fibers, pellets, and thin films because the characteristics of a single CNT can be used on a macroscopic scale to fabricate reliable, high-performance devices $[8,9]$. BP is a macroscopic assembly of nanotubes that are formed through filtration after their dispersion in organic solvents. BP typically has a laminar structure with a random orientation of the bundles of tubes in the plane of the sheet [10]. High structural integrity in this MWCNT BP is thought to be derived primarily from their long lengths (up 
to micrometer scale), because the longer the tubes, the greater the mechanical robustness of the intermingled bundle. There has been a considerable level of interest in developing novel BP-based composite materials with improved mechanical properties and electrical conductivity for numerous potential applications. It has been established that SWCNTs exhibit considerably high electrical conductivity at room temperature because the charge carrier in the nanotubes exhibits substantial mobility [11]. CNTs exhibit excellent TE properties because of their nanoscale, low-dimensional, and porous structures [12]. Several studies have reported that the values of Seebeck coefficients for individual types of SWCNTs and MWCNTs are approximately $42 \mu \mathrm{V} / \mathrm{K}[13]$ and $82 \mu \mathrm{V} / \mathrm{K}$ [14], respectively, and for SWCNT and MWCNT films range from 20 to $25 \mu \mathrm{V} / \mathrm{K}[15,16]$. Zhan et al. [17] showed that the TE efficiency of SWCNTs could be improved by fabricating SWCNT/ceramic nanocomposites with metal oxides such as $\mathrm{Al}_{2} \mathrm{O}_{3}$ and nanocrystalline $\mathrm{Y}_{2} \mathrm{O}_{3}$-stabilized tetragonal $\mathrm{ZrO}_{2}$ polycrystals.

A review of the extant literature shows that few reports have focused on improving the thermoelectric properties of MWCNT-based BP and there is no report on the effect of the deposition of metal alloy nanoparticles on MWCNT BP. The purpose of this study was to elucidate the TE performance of MWCNT BP by depositing Bi, Te, and Sb alloys on the surface of $\mathrm{BP}$ and to measure the corresponding electrical resistivity and Seebeck coefficients. A thermal evaporation method was used because it guarantees high degree of homogeneity and uniform coverage. This report provides a simple and convenient method for fabricating high-quality and flexible MWCNT BP that exhibits promising electrical properties.

\section{Materials and Methods}

2.1. Fabrication of MWCNT Buckypaper. The BP used in this study was fabricated using MWCNTs through vacuum filtration. The modified procedure used for fabricating the MWCNT BP was adopted from previous reports $[18,19]$. The MWCNTs were dispersed in distilled water and stabilized using Triton-X-100, a nonionic surfactant. The aqueous suspension of MWCNTs was produced using ultrasonication (Misonic, $20 \mathrm{kHz}, 63 \mathrm{~W}$ ). Following the filtration and drying processes, the paper was peeled carefully and washed in an isopropyl alcohol bath to completely remove the surfactant, thereby producing a thin sheet (approx. $110 \mu \mathrm{m}$ ) of random network MWCNT BP (RBP). Compared with aligned membranes, the fabricated RBP exhibited a broader distribution of larger pores, which offers several advantages. For example, it can be rolled or bent without cracking, which is a unique mechanical property that is superior to that of the conventional inorganic TE materials applied in flexible energy conversion systems.

2.2. Deposition of Metal Alloys. A thermal evaporation technique was used to deposit the TE metal alloy on the RBP surface. The metal films (approx. $80 \mu \mathrm{m}$ ) were deposited onto the BP by Bi, Sb, and Te granules (99.99\%) as a vapor source. The BP substrate was fixed on a ceramic holder and
TABLE 1: EDX-weight percentage of the elements present in the BP samples.

\begin{tabular}{lcccc}
\hline Elements $^{*}$ & BiTe-RBP & SbBi-RBP & SbTe-RBP & SbBiTe-RBP \\
\hline $\mathrm{C}$ & 58.26 & 55.22 & 54.40 & 48.12 \\
$\mathrm{O}$ & 6.15 & 5.35 & 4.77 & 3.20 \\
$\mathrm{Bi}$ & 7.18 & 10.30 & - & 10.22 \\
$\mathrm{Sb}$ & - & 14.58 & 18.07 & 17.67 \\
$\mathrm{Te}$ & 16.45 & - & 6.65 & 12.15 \\
\hline
\end{tabular}

${ }^{*} \mathrm{Fe}, \mathrm{Ti}$, and $\mathrm{Al}$ are the impurities present in the sample due to the ultrasonication tip.

inserted into a deposition chamber that was subsequently evacuated under a relative pressure of $10^{-5}$ torr. The distance from source to the substrate was approximately $30 \mathrm{~cm}$. The total deposition time varied from 1 to $2 \mathrm{~min}$ in a vacuum. The ratio of the metal source was $1: 1$ ( $0.06 \mathrm{~g}$ each). The samples after deposition of alloy of $\mathrm{Bi}+\mathrm{Te}, \mathrm{Sb}+\mathrm{Bi}, \mathrm{Sb}+\mathrm{Te}$, and $\mathrm{Sb}+$ $\mathrm{Bi}+\mathrm{Te}$ onto the RBP, surface were referred as BiTe-RBP, SbBi$\mathrm{RBP}, \mathrm{SbTe}-\mathrm{RBP}$ and $\mathrm{SbBiTe}-\mathrm{RBP}$, respectively.

2.3. Characterizations. The surface morphology and composition analyses of the BP were examined using field emission scanning electron microscopy (FESEM) with energy dispersive X-ray (EDX) JEOL JSM-7000F. The temperaturedependent electrical resistivity $(\rho)$ measurements of the BP samples were conducted using a standard programmable DC voltage/current detector four-point probe method in a temperature range from 300 to $550 \mathrm{~K}$. Direct thermal conductivity of the pristine RBP at $300 \mathrm{~K}$ was measured using a hot disk analyzer TPS 2500. The Seebeck coefficient (S) was determined by applying a temperature gradient along the sample and measuring the thermoelectric voltage. For all the samples, electrical resistivity $(\rho)$ and Seebeck coefficient $(S)$ were measured as a function of temperature, and $S(T)$ was measured for 3 and 5 sheets of the metal-deposited BP samples which were connected in series.

\section{Results and Discussion}

3.1. Morphological and Compositional Analysis. Figures 1(a)1(e) show the FESEM images of the RBP, BiTe-RBP, SbBi-RBP, $\mathrm{SbTe}-\mathrm{RBP}$, and SbBiTe-RBP, respectively. The microstructures of all samples illustrate the random entanglement, and the network structures of CNTs were formed. Figure 1(a) shows the porous structure of RBP, indicating the complete removal of surfactants between the tubes [20], whereas Figures 1(b)-1(d) show an increase in the tube diameter of metal alloy/MWCNT composites. It reveals that the deposition of metal nanoparticles on the surface caused a densely packed network structure of CNTs in the buckypaper. Furthermore, the network structures of the MWCNT BP were unaltered by the deposition of the metal particles. The insets in Figures 1(a)-1(e) are images of the pure and metal-deposited BP samples. Table 1 shows the elemental composition of the metal-deposited BP samples observed using EDX. 


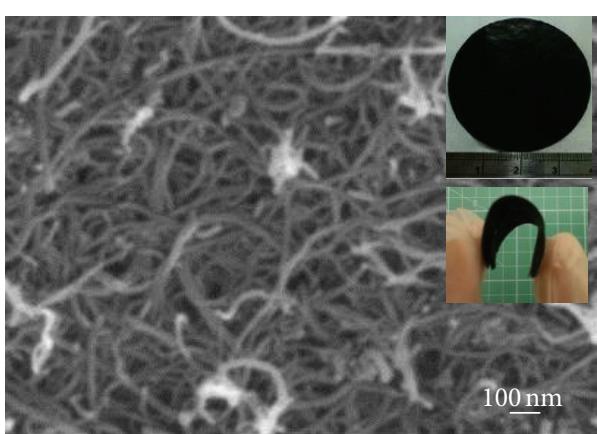

(a)

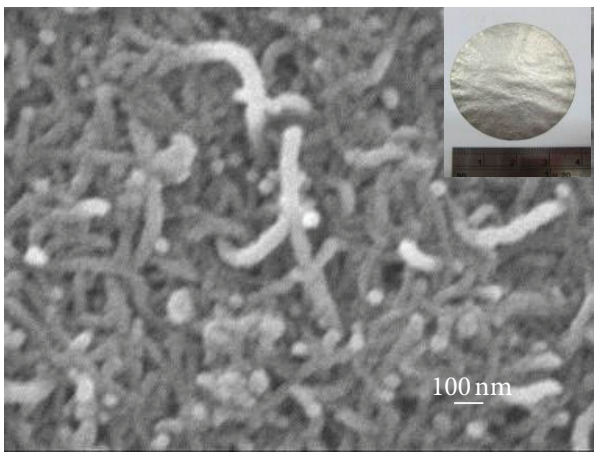

(c)

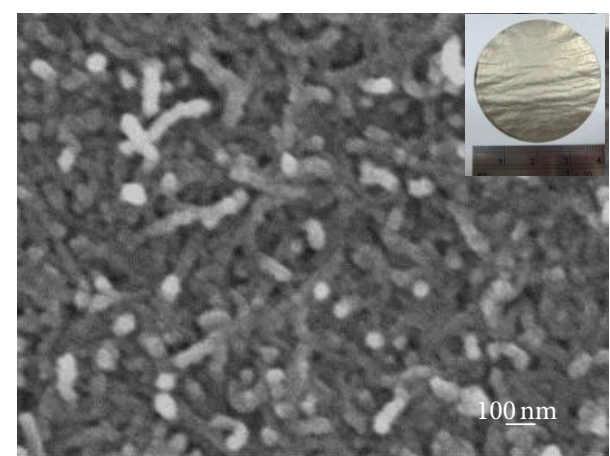

(b)

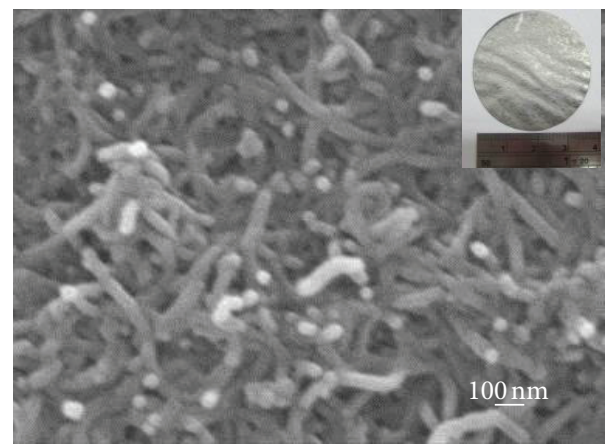

(d)

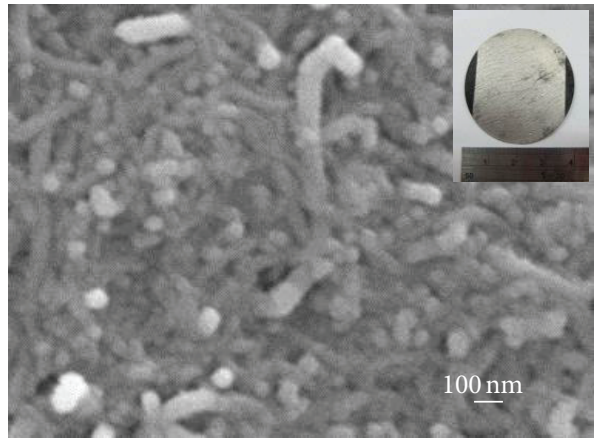

(e)

Figure 1: FE-SEM images of buckypaper samples for (a) RBP, (b) BiTe-RBP, (c) SbBi-RBP, (d) SbTe-RBP, and (e) SbBiTe-RBP. Insets in (a)-(e) are the photo images of buckypaper samples, respectively.

3.2. Electrical Resistivity Studies. Figure 2 shows the hightemperature electrical resistivity $(\rho)$ plots for the pure and metal-deposited MWCNT-BP samples. The room temperature (RT) resistivity for all samples ranged from approximately 0.01 to $0.06 \Omega-\mathrm{cm}$, which is comparatively low and decreases as the temperature increased, indicating that all the BP samples exhibited strong semiconducting behavior. The difference in the observed resistivity among the various metal alloy-deposited BP were attributed to the oxidation of nanotubes on the surface of buckypaper [17]. The increased slope indicated that the localization length decreased as the barriers between the intertube contacts increased and the oxidation is due to absorption of moisture on the surface of the $\mathrm{BP}$ during the measurement process [20]. These results show that the temperature dependence of the resistivity for all the samples was mostly dominated by the transportation of carriers between the tube junctions through hopping or tunneling, thereby resulting in semiconducting behavior [21, 22].

Based on the observed semiconducting behavior, the variable-range hopping $(\mathrm{VRH})$ model appears to be the predominant conduction mechanism [23]. It could be considered that the BP is a 3-dimensional network of dispersed bundle-bundle junctions that allow the carriers to move within a sheet. Therefore, the electrical conduction occurs either in the tube-tube, within the nanotube bundles or between neighbour bundles through their contacts. The resistivity is primarily dominated by the electric field gradient across the material because the electrons must hop between different bundles of CNTs [23]. The essential part is that the electrons hopped between conducting entities (NTs) were separated by the metallic particles which leads to 


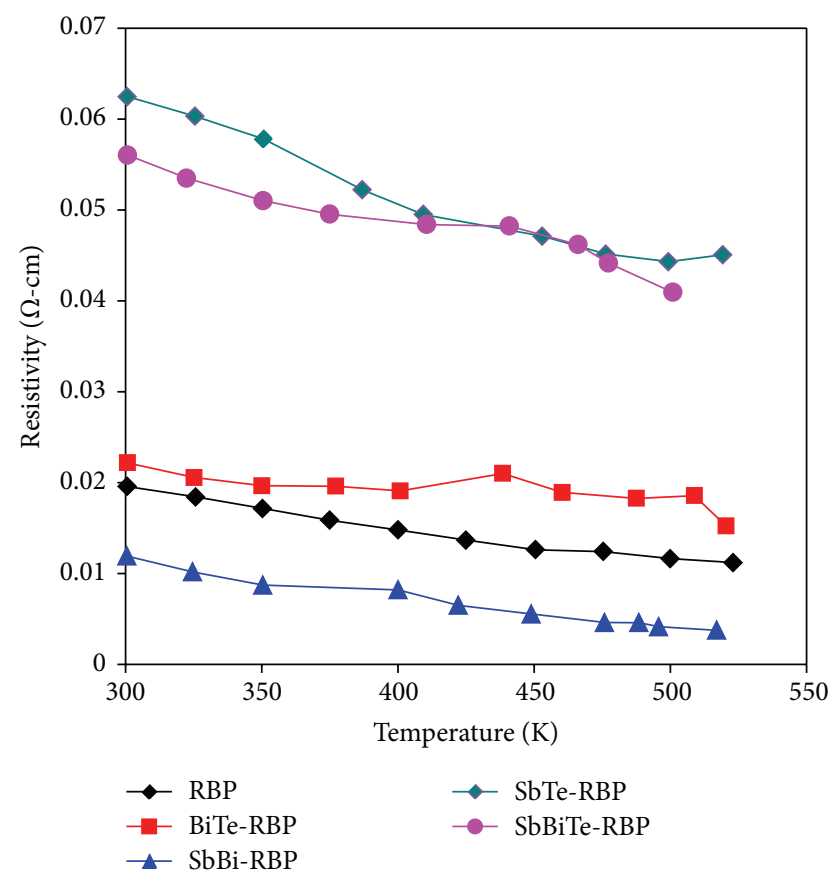

FIGURE 2: High-temperature-dependent electrical resistivity measurements for pure and metal alloy-deposited RBP samples.

a nonmetallic dependence of $\rho(T)$. The hopping conduction was probably the characteristic electric transport behavior between the nanotubes and bundles.

Compared with the pure samples, the metal-deposited RBP samples exhibited an increased resistivity value, indicating that the particles deposited onto the surface of the nanotubes might have caused a slight blockage at the intertube junctions and thereby forming a barrier for the hopping and tunneling of electrons. Kim et al. [24] also reported that the presence of metal content within the CNTs can affect the RT resistivity. Previous research indicated that the electrical conductivity of fully connected CNT networks can be limited by tube-tube contact resistance [25].

3.3. Seebeck Coefficient Measurement. Figure 3(a) shows the temperature-dependent Seebeck coefficient $S(T)$ for a single sheet of metal-deposited MWCNT buckypaper. Figures 3(b) and 3(c) show the Seebeck coefficients for 3 and 5 sheets of metal-deposited BP connected in series, respectively. The Seebeck coefficient is related to the transport of energetic charged particles, whereas the electrical conductivity is related to the transport of all mobile charges [26]. In this study, the observed $S(T)$ values were negative, indicating that the majority of carriers were electrons. For all samples, the strongly curved T-dependence of " $S$ ", which exhibited a nonlinear behavior, shows the semiconducting nature of the CNT networks, which also supports the resistivity results [27].

Table 2 shows the maximum $S(T)$ values obtained for the single layer as well as for the 3 and 5 sheets in series. The $S(T)$ value for the single layer of pristine MWCNT BP was $-13.49 \mu \mathrm{V} / \mathrm{K}(\kappa=0.1824 \mathrm{~W} / \mathrm{mK}$ at $300 \mathrm{~K})$ is comparable $[15,23,28]$, and in our, case it increases to approximately
TABLE 2: Measured Seebeck coefficient $S(-\mu \mathrm{V} / \mathrm{K})$ for metal alloydeposited RBP samples.

\begin{tabular}{lccc}
\hline Samples & Single & 3 sheets & 5 sheets \\
\hline BiTe-RBP & 20.66 & 49.44 & 67.22 \\
SbBi-RBP & 22.15 & 45.22 & 77.98 \\
SbTe-RBP & 20.17 & 41.31 & 65.28 \\
SbBiTe-RBP & 19.90 & 47.14 & 74.89 \\
\hline
\end{tabular}

$22( \pm 1) \mu \mathrm{V} / \mathrm{K}$ after the deposition of TE metal alloys. The variation in the Seebeck coefficient originated from the change in the doping of metal alloys and its composition on MWCNTs and the amount of charges transferred to the BP. It was also stated that the Seebeck coefficient for CNT mats is independent of the CNT type, and high energetic charges are transported regardless of the junction types [28]. This unique relationship between the Seebeck coefficient $(S)$ and high electric conductivity $(\sigma)$ implies that it is possible to balance the metal alloy films on the surface of BP to increase the carrier mobility and attain the maximum power factor $\left(S^{2} \sigma\right)$. We also demonstrated that 3 and 5 layers of metaldeposited BP can be used to attain a high Seebeck coefficient of approximately $50( \pm 4)$ and $75( \pm 6) \mu \mathrm{V} / \mathrm{K}$, respectively. This shows that an array of metal-deposited BP connected in series can achieve high " $\sigma$ " and $S^{2} \sigma$, thereby enhancing the thermoelectric efficiency.

We determined that increasing the thickness of the metal alloys on the BP surface weakens the quantum confinement effects and reduces the interaction between the metal particles and CNTs. This reduces the carrier density in the BP, which increases the resistivity of the sample. Furthermore, the intertube junctions in the BP are essential factors for improving the electrical transport and mechanical strength. This work demonstrates the fundamental challenges associated with employing the TE nanoparticles within BP to increase the surface properties as well as the inter-particle contacts, which was primarily considered in order to maximize the TE efficiency. We are further involved in controlling various parameters such as composition and thickness to achieve higher electrical and low thermal conductivity for processing the MWCNT-based BP as efficient thermoelectric (TE) material into a functional device.

\section{Conclusion}

In summary, the alloy of $\mathrm{Bi}, \mathrm{Sb}$, and $\mathrm{Te}$ nanoparticles was deposited on the surface of the buckypaper by employing a thermal evaporation method. The electrical measurements of the pure and metal alloy-deposited MWCNT buckypapers have been performed. The RT electrical resistivity was observed in the range from 0.01 to $0.06 \Omega-\mathrm{cm}$, which also demonstrated the semiconductivity of the CNT networks. The observed $S(T)$ values are comparable to those of BPs synthesized using various methods. An increase in the value was observed for the Seebeck coefficient to approximately 50 and $75 \mu \mathrm{V} / \mathrm{K}$ by connecting the metal alloy-deposited BP sheets in series. The results show the potential of these TE NPs to be further refined and maximized to increase the efficiency 


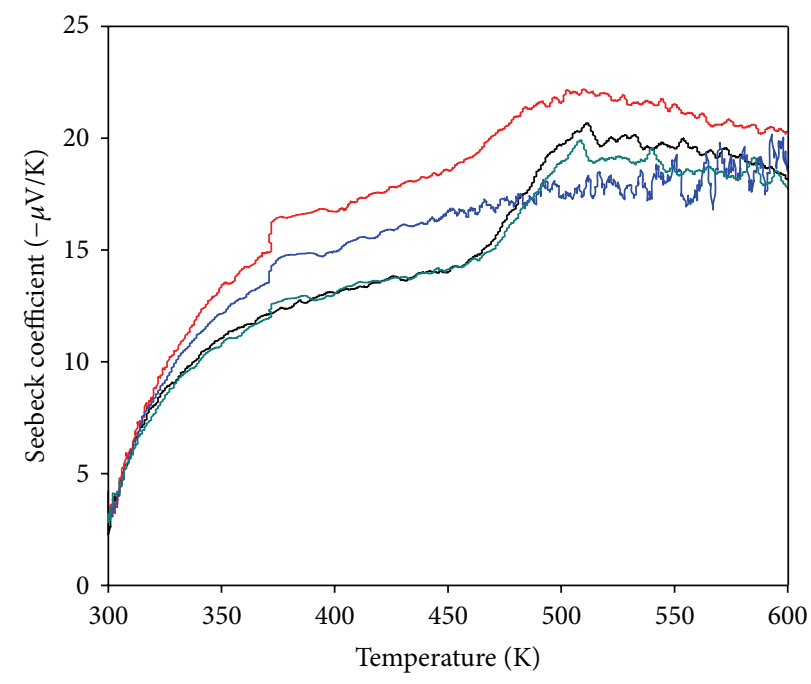

(a)

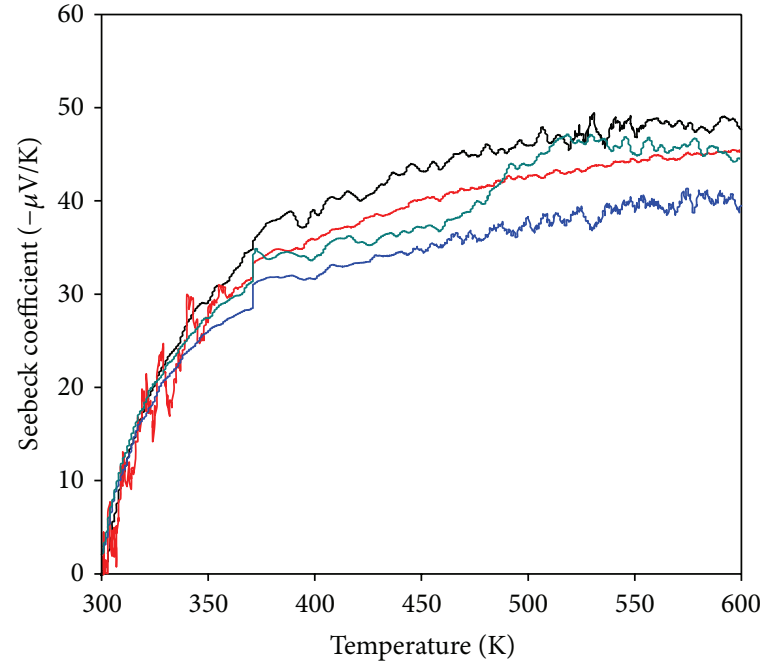

(b)

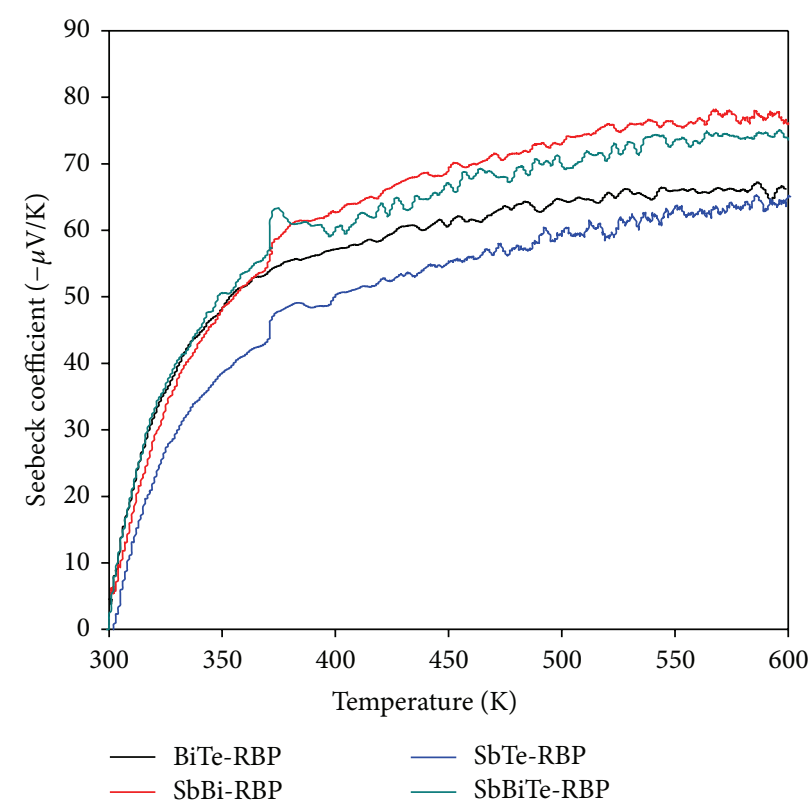

(c)

FIGURE 3: Seebeck coefficient $S(T)$ for metal alloy-deposited RBP samples. (a) Single layer, (b) 3 sheets connected in series, and (c) 5 sheets connected in series.

of BP-based thermoelectric materials. Further study on the inclusion of these TE particles in BP by controlling their size and composition to maximize the inter-tube contacts and improve surface properties through sample processing, to increase the electrical conductivity and enhance the thermoelectric efficiency, is part of our ongoing studies in this field.

\section{Acknowledgments}

This work was supported by the Global Research and Education on Environment and Society (GREEnS) program, Tunghai University, Taiwan, and the National Science Council, Taiwan (NSC101-2221-E-029-006 and NSC101-2221-E-029010).

\section{References}

[1] W. Liu, X. Yan, G. Chen, and Z. Ren, "Recent advances in thermoelectric nanocomposites," Nano Energy, vol. 1, no. 1, pp. 42-56, 2012.

[2] M. S. Dresselhaus, G. Chen, M. Y. Tang et al., "New directions for low-dimensional thermoelectric materials," Advanced Materials, vol. 19, no. 8, pp. 1043-1053, 2007.

[3] A. L. Prieto, M. Martín-González, J. Keyani, R. Gronsky, T. Sands, and A. M. Stacy, "The electrodeposition of high-density, ordered arrays of $\mathrm{Bi}_{1-x} \mathrm{Sb}_{x}$ nanowires," Journal of the American Chemical Society, vol. 125, no. 9, pp. 2388-2389, 2003.

[4] D. Mott, N. T. Mai, N. T. B. Thuy et al., "Bismuth, antimony and tellurium alloy nanoparticles with controllable shape and 
composition for efficient thermoelectric devices," Physica Status Solidi (A), vol. 208, no. 1, pp. 52-58, 2011.

[5] H. J. Goldsmid, Semiconductors and Semimetals, vol. 69, chapter 1, Academic Press, New York, NY, USA, 2000.

[6] W. J. Xie, X. F. Tang, Y. G. Yan, Q. J. Zhang, and T. M. Tritt, "High thermoelectric performance BiSbTe alloy with unique low-dimensional structure," Journal of Applied Physics, vol. 105, no. 11, Article ID 113713, 2009.

[7] Y. Zhang, X. L. Wang, W. K. Yeoh, R. K. Zeng, and C. Zhang, "Electrical and thermoelectric properties of single-wall carbon nanotube doped $\mathrm{Bi}_{2} \mathrm{Te}_{3}$," Applied Physics Letters, vol. 101, no. 3, Article ID 031909, 4 pages, 2012.

[8] J. Liu, J. Sun, and L. Gao, "Flexible single-walled carbon nanotubes/polyaniline composite films and their enhanced thermoelectric properties," Nanoscale, vol. 3, no. 9, pp. 3616-3619, 2011.

[9] W. Zhao, S. Fan, N. Xiao et al., "Flexible carbon nanotube papers with improved thermoelectric properties," Energy and Environmental Science, vol. 5, no. 1, pp. 5364-5369, 2012.

[10] L. Berhan, Y. B. Yi, A. M. Sastry, E. Munoz, M. Selvidge, and R. Baughman, "Mechanical properties of nanotube sheets: alterations in joint morphology and achievable moduli in manufacturable materials," Journal of Applied Physics, vol. 95, no. 8, pp. 4335-4345, 2004.

[11] T. W. Ebbesen, H. J. Lezec, H. Hiura, J. W. Bennett, H. F. Ghaemi, and T. Thio, "Electrical conductivity of individual carbon nanotubes," Nature, vol. 382, no. 6586, pp. 54-56, 1996.

[12] I. Kunadian, R. Andrews, M. P. Mengüç, and D. Qian, "Thermoelectric power generation using doped MWCNTs," Carbon, vol. 47, no. 3, pp. 589-601, 2009.

[13] C. H. Yu, L. Shi, Z. Yao, D. Li, and A. Majumdar, "Thermal conductance and thermopower of an individual single-wall carbon nanotube," Nano Letters, vol. 5, no. 9, pp. 1842-1846, 2005.

[14] P. Kim, L. Shi, A. Majumdar, and P. L. McEuen, "Thermal transport measurements of individual multiwalled nanotubes," Physical Review Letters, vol. 87, no. 21, Article ID 215502, 4 pages, 2001.

[15] C. Meng, C. Liu, and S. Fan, "A promising approach to enhanced thermoelectric properties using carbon nanotube networks," Advanced Materials, vol. 22, no. 4, pp. 535-539, 2010.

[16] M. T. Byrne and Y. K. Guin'Ko, "Recent advances in research on carbon nanotube-polymer composites," Advanced Materials, vol. 22, no. 15, pp. 1672-1688, 2010.

[17] G. D. Zhan, J. D. Kuntz, A. K. Mukherjee, P. Zhu, and K. Koumoto, "Thermoelectric properties of carbon nanotube/ ceramic nanocomposites," Scripta Materialia, vol. 54, no. 1, pp. 77-82, 2006.

[18] Z. Wang, Z. Liang, B. Wang, C. Zhang, and L. Kramer, "Processing and property investigation of single-walled carbon nanotube (SWNT) buckypaper/epoxy resin matrix nanocomposites," Composites A, vol. 35, no. 10, pp. 1225-1232, 2004.

[19] Y. W. Chen, H. Y. Miao, M. Zhang, R. Liang, C. Zhang, and B. Wang, "Analysis of a laser post-process on a buckypaper field emitter for high and uniform electron emission," Nanotechnology, vol. 20, no. 32, Article ID 325302, 2009.

[20] J. G. Park, J. Smithyman, C. Y. Lin et al., "Effects of surfactants and alignment on the physical properties of single-walled carbon nanotube buckypaper," Journal of Applied Physics, vol. 106, no. 10, Article ID 104310, 6 pages, 2009.
[21] A. B. Kaiser, G. Düsberg, and S. Roth, "Heterogeneous model for conduction in carbon nanotubes," Physical Review B, vol. 57, no. 3, pp. 1418-1421, 1998.

[22] J. C. Charlier, "Defects in carbon nanotubes," Accounts of Chemical Research, vol. 35, no. 12, pp. 1063-1069, 2002.

[23] A. M. Díez-Pascual, J. Guan, B. Simard, and M. A. GómezFatou, "Poly(phenylene sulphide) and poly(ether ether ketone) composites reinforced with single-walled carbon nanotube buckypaper: II-mechanical properties, electrical and thermal conductivity," Composites A, vol. 43, no. 6, pp. 1007-1015, 2012.

[24] H. M. Kim, K. Kim, C. Y. Lee et al., "Electrical conductivity and electromagnetic interference shielding of multiwalled carbon nanotube composites containing Fe catalyst," Applied Physics Letters, vol. 84, no. 4, pp. 589-591, 2004.

[25] A. Buldum and J. P. Lu, "Contact resistance between carbon nanotubes," Physical Review B, vol. 63, no. 16, Article ID 161403(R), 4 pages, 2001.

[26] C. Yu, Y. S. Kim, D. Kim, and J. C. Grunlan, "Thermoelectric behavior of segregated-network polymer nanocomposites," Nano Letters, vol. 8, no. 12, pp. 4428-4432, 2008.

[27] J. Hone, I. Ellwood, M. Muno et al., "Thermoelectric power of single-walled carbon nanotubes," Physical Review Letters, vol. 80, no. 5, pp. 1042-1045, 1998.

[28] Y. J. Choi, Y. Kim, S. G. Park et al., "Effect of the carbon nanotube type on the thermoelectric properties of CNT/Nafion nanocomposites," Organic Electronics, vol. 12, no. 12, pp. 21202125, 2011. 

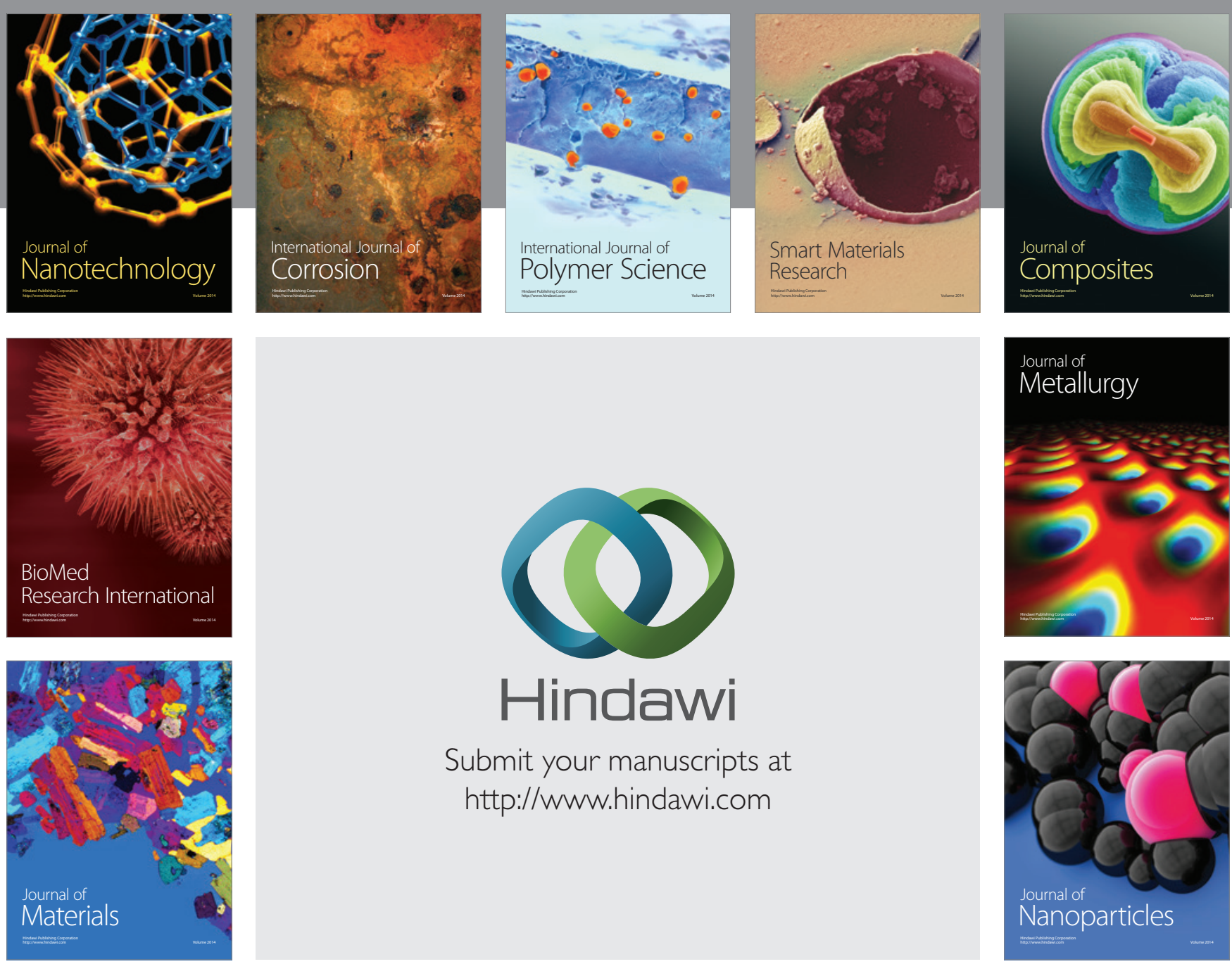

Submit your manuscripts at http://www.hindawi.com
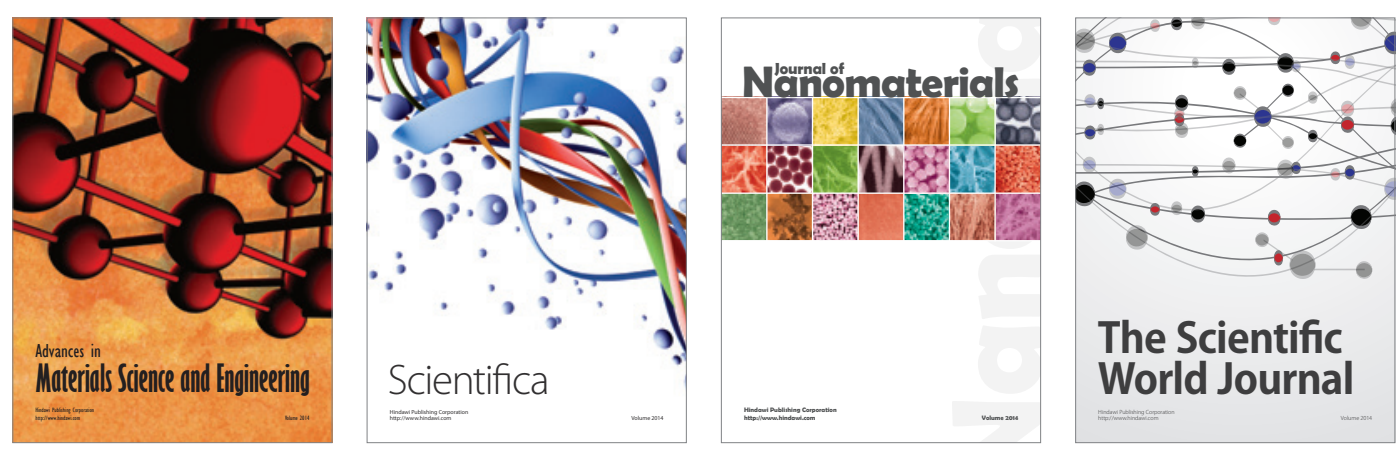

\section{The Scientific World Journal}
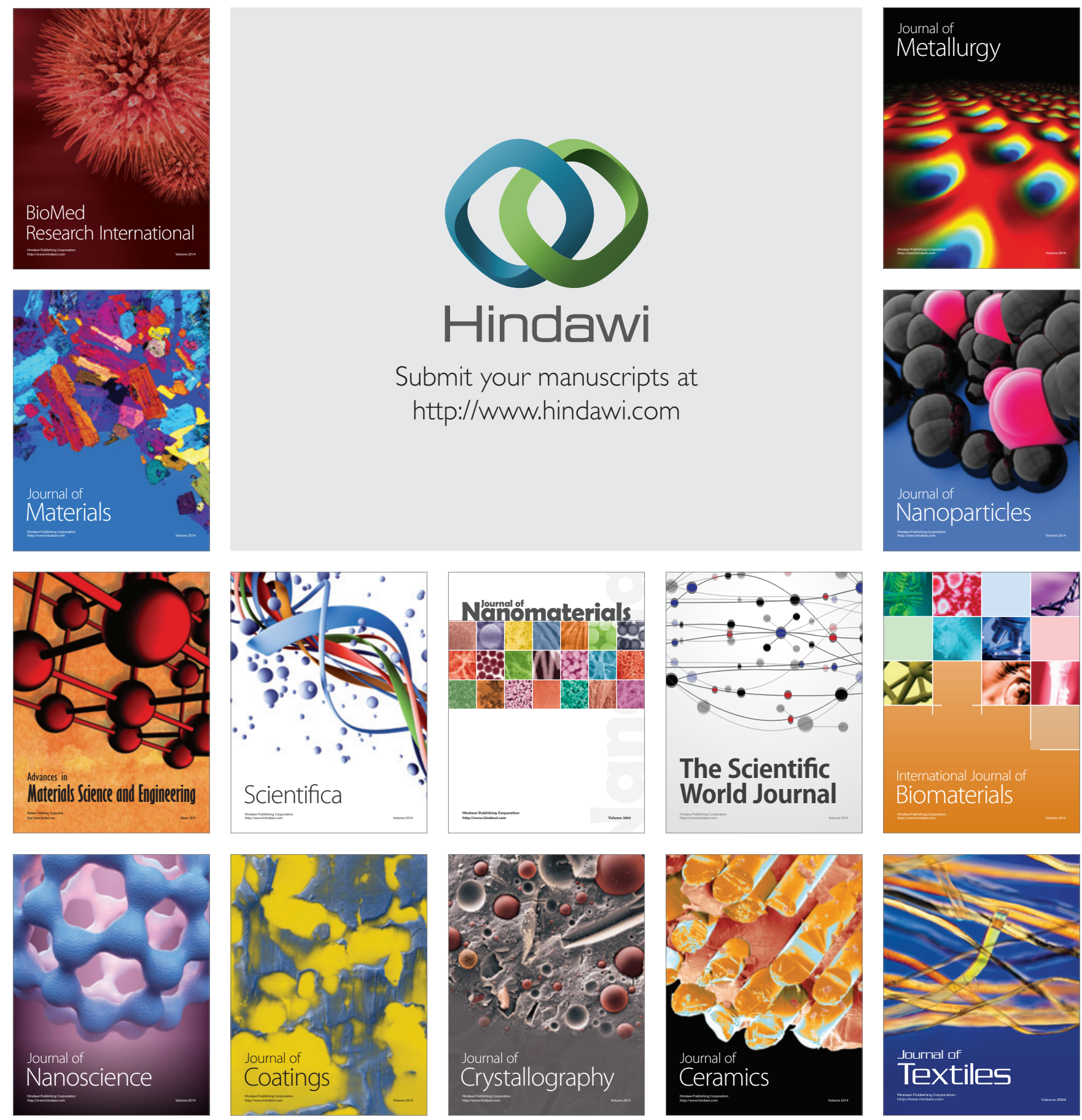